\title{
$\$$ sciendo
}

Athanasios Despotis (ed.), Participation, Justification and Conversion, Eastern Orthodox Interpretation of Paul and the Debate between "Old and New Perspective on Paul”. WUNT 2/442, Verlag Mohr Siebeck, Tübingen 2017, $435 \mathrm{~S}$.

Hans Klein ${ }^{*}$

Im Vorwort dieses Bandes stellt der Herausgeber dies Sammelwerk von Studien als Folge seiner Habilitationsschrift über „Die New Perspective on Paul und die griechisch orthodoxe Paulusinterpretation" (2014) vor. Es ist ein Projekt, das von der Deutschen Forschungsgemeinschaft subventioniert wurde. Gedacht ist es ist als Festschrift zum 180. Bestehen der Theologischen Fakultät an der Universität von Athen (2017) und gleichzeitig als Gruß für den General Meeting der SNTS in Athen 2018. Es enthält Beiträge aus Griechenland, Rumänien, Deutschland, Südafrika und den USA.

In der Einleitung stellt der Herausgeber sämtliche Studien seiner Autoren vor, vergleicht sie streckenweise miteinander und erarbeitet somit den Gewinn der New Perspective für das Gespräch mit der orthodoxen Theologie. Diese Darstellung ist für den außenstehenden Theologen darum wichtig, weil er darin auf Dinge zu achten angehalten wird, die sich von außen gesehen nicht direkt ergeben.

Michael Wolter, „,The Realty of Faith: Some Thoughts about the Significance of Faith in the Theology of Paul" (S. 13-27) geht in einer vermutlich aus anderem Anlass geschriebenen Studie dem Verständnis des Glaubens und der Glaubensrealitäten aufgrund der Aussagen von 1 Thess 2,13 nach. Er beginnt mit der Feststellung, dass die Glaubenden einer Gruppe angehören, die sie von anderen Gruppen unterscheiden. Sie hören und verinnerlichen die Botschaft, glauben. Damit legen sie die Botschaft für sich selber aus. Die Botschaft von Jesu Auferstehung und seinem Tod uns zum Heil ist eine Glaubensaussage, die eine neue Realität schafft, eine Glaubensrealität, die sich von den Dingen dieser Welt, Paulus sagt „nach dem Fleisch“ unterscheidet. So ist die Wendung „durch Christus“ mit jener „durch den Glauben“ austauschbar. Und auf diese Weise kann Paulus auch vom „Glauben Jesu Christi“ sprechen, der nicht mit Treue Jesu zu Gott wiedergegeben werden kann. Denn auch die Redeweise von der Treue Jesu setzt Glauben voraus.

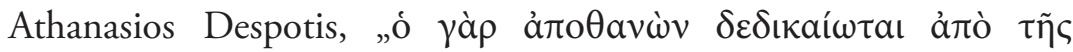

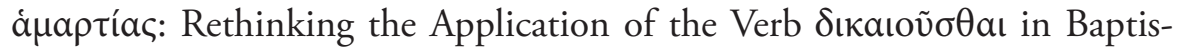
mal Contexts from the Perspective of Rom 6:7“ (S. 29-57) untersucht in

\footnotetext{
* Hans Klein, Prof. em. Dr. Theol., Evangelische Fakultät der Universität Lucian Blaga Sibiu, Str. Negoi, Nr. 47, Sibiu; e-mail: hansheideklein@gmail.com.
} 
methodisch vorzüglicher Weise ein scheinbares Nebenproblem der Rechtfertigungslehre des Paulus und fragt, wie das Absterben der Sünde bei der Taufe zu verstehen sei. Dazu zieht er Texte aus dem NT und der biblischen wie hellenistischen Welt heran. Von 1 Kor 6,11 her ergibt sich ihm die enge Verbindung von Taufe, Geistesgabe und Rechtfertigung. Nach Röm 6,17 sündigt der Christ nicht mehr, das meint, dass die Sünde ihn nicht mehr dominiert. Er ist gerechtfertigt durch den Tod in der Taufe, von der Macht der Sünde befreit. Man kann nach Despotis nicht wirklich zwischen der gegenwärtigen und der eschatologischen "Rechtfertigung" trennen, es geht um einen fortschreitenden Prozess, einen Weg zur Vollendung. Aber in Röm 5,9 macht Paulus einen Unterschied zwischen gegenwärtiger Rechtfertigung und künftigem Heil. Vielleicht wäre ein Hinweis darauf hilfreich gewesen, dass der Getaufte nur noch „Fehler“ ( $\pi \alpha \rho \alpha \pi \tau \dot{\omega} \mu \alpha \tau \alpha)$ macht (Gal 6,1).

Jacobus (Kobus) Kok and John Anthonny Dunne, „Participation in Christ and Missional Dynamics in Galatians" (S. 59-85) hebt für die Begründung der Partizipation mit Christus den Bericht des Paulus über seine Bekehrung in Gal 1,15 hervor und betont, dass Christus „in “ihm offenbar wurde. Es war aber kein mystisches Erlebnis. Sein Ich ist dabei nicht in Christus aufgegangen. Gal 2,20 lehrt, dass Christus in ihm ist, aber er lebt weiterhin auf dieser Welt, auch wenn er ihr nach Gal 1,4 geistlich enthoben ist. Als solcher soll sich der Christ in dieser Welt bewähren und Früchte des Geistes aufweisen können, die in Gal 5,22f aufgezählt werden. Der Dienst des Glaubenden gilt allen Menschen, aber besonders den Mitgliedern der Kirche (Gal 6,10).

Kontstantinos Nikolakopoulos, „Paulus über «Gerechtigkeit» und «Rechtfertigung». Exegetische Perspektiven unter Berücksichtigung von 1 Kor 1,30“ (S. 89-106) spricht von mehreren neuen Perspektiven, die Horizonte eröffnen können und nicht die Sichtweise reduzieren sollten. Paulus stellt seine Bildung im Judentum und Hellenismus in den Dienst des Evangeliums. Dafür analysiert Nikolakopoulos die kurze Aussage über Christus, der zur Gerechtigkeit/ Rechtfertigung geworden ist, in ihrem Kontext und zieht Parallelen aus Röm und Gal heran. Er versteht die Rechtfertigung als Qualifizierung des Menschen durch Gott, die im Glauben angeeignet wird. Seine Erkenntnis formuliert N. in dem Satz: „Die erlösende Wirkung Jesu Christi ...schafft eine dynamische Wirklichkeit, die der Glaubende bereits innerhalb der Kirche durch den Heiligen Geist real erlebt."

Stelian Tofană, „Treasure in Earthen vessels..." (2 Cor 4,7-11) (S. 107-123) sieht in dem „Schatz“ das „Licht des Evangeliums“, das die Herzen derer wandelt, die glauben und ihre Hoffnung auf Gott setzen. Dieser Schatz führt zur apostolischen Mission, die Leiden mit sich bringt, ein Leiden, das, 
wie er mit einem Zitat aus Chrysostomos belegt, zum neuen Leben führt. Dafür hätte auch 4,16 angeführt werden können, ein Text, den er auf S. 112 nur tangentiell erwähnt. Aufgrund der Forderung Dunns, dass die Theologie des Paulus „hinter den Aussagen“ erschaut werden muss, ergibt sich für Tofană, dass der geistliche Mensch auf dem Weg vom „alten“ zum „neuen“ Menschen, zur neuen Kreatur ist, auf dem Weg der Nachfolge Christi vom Leiden zur Herrlichkeit, in einem Prozess der Heiligung, der Theosis.

Edith M. Humphrey, „The Potency of the New Creation in the World (2 Cor 5:16-21) “ (S. 125-157) stellt den Abschnitt zunächst in den größeren Zusammenhang von 2 Kor 2,14-7,4, der von der Herrlichkeit des apostolischen Amtes, von der Verherrlichung der Christen, vom Sichtbaren und Unsichtbaren und von der eschatologischen Zukunft vor dem zu behandelnden Abschnitt spricht, dem wiederum ethische Ermahnungen folgen. Für Humphrey hängen diese Dinge alle zusammen: Versöhnung und neue Schöpfung, Verwandlung in dieser Welt auf die künftige zu und rechtes Verhalten der Christen. Sie stellt fest, dass das von Luther ausgehende Konzept einer geschenkten Gerechtigkeit zu sehr passiv gefasst ist und, was auch die New Perspective bemängelt, die „Werke der Gerechtigkeit“ neu versteht, aber vom orthodoxen Verständnis her ist die Verwandlung des Christen zur Verkörperung der Gerechtigkeit Gottes in Jesus Christus maßgebend, eine Wandlung, die die gesamte Person betrifft.

Vasile Mihoc, „Galatians 2,15-21 a Commentary Challenging the «New Perspective on Pal»" (S. 159-178) legt eine minutiöse Exegese von Gal 2,15-21 vor. Er versteht diesen Abschnitt als Wiedergabe der öffentlichen Worte des Paulus, die dieser an Petrus, Barnabas und andere Christen in Antiochia richtete. Vom Abschluss dieser Perikope her ergibt sich bei Mihoc, dass bei Paulus bei seinem Damaskuserlebnis das Eindringen des Christus in den Apostel erfolgt ist, sodass Christus hinfort sein Leben bestimmt. Rechtfertigung ist somit nicht juristisch, als Proklamation zu verstehen, sondern als ein von Gott durch den Glauben erfolgtes Geschehen, als Eindringen des Christus in das Innere des Apostels, mit der Bekehrung und Taufe gegeben. Damit beginnt das neue Leben in Christus. Auf das Anliegen der „New Perspective" - die neue Beurteilung dessen, wie Werkgerechtigkeit zu sehen ist - geht M. nicht ein. Der Herausgeber stellt diesen Mangel fest. Die angeführte Literatur ist weitgehend älteren Datums.

Sotirios Despotis, „Eine östlich-orthodoxe Lektüre von Gal 3,69.23-29“ (S. 181-200) sieht in der Hervorhebung der Ekklesiologie eine Neue Perspektive. Er erkennt, dass Gal 3,23-29 der Tradition, konkret der urchristlichen Taufliturgie, entnommen ist, was ihn veranlasst, Rechtfertigung und Taufe und damit die Eingliederung in die Gemeinde zusammen 
zu sehen. Diese Rechtfertigung geschieht nicht aufgrund von Werken des Gesetzes (Beschneidung und Feste), sondern durch die Einhaltung des Bundes mit dem Gottesvolk. Die Thora ist nicht die Unterweisung des liebenden Vaters, sie hat nur die Funktion des Pädagogen und des Wächters (das entspricht dem usus pedagogocus der Reformatoren). Mit den Kirchenvätern verweist Despotis auf die Opferung Isaaks als Zeichen des Glaubens, den er als Annahme des Evangeliums und als Treue kennzeichnet. Glaube, Bekehrung und Taufe werden zusammen gesehen. Hier wird Rechtfertigung erlebt, die gleichzeitig in die Sohnschaft führt und eine neue Kreatur bewirkt. Damit wird eine dynamische Erfahrung ausgelöst, die „Früchte des Geistes“ hervorbringt und hervorbringen soll.

Jack Khalil, „An Interpretation of Rom 3:2-26 within Its Proper Context" (S. 201-241) widmet sich einer ausführlichen Besprechung von Röm 3,21-25, indem er zunächst den Abschnitt 3,1-20 bedenkt, wonach Paulus alle Menschen aufgrund der Aussagen der Schrift zu Sündern erklärt. Dieselben weissagen Gottes Gerechtigkeit, die als eine Gott eigene, von ihm ausgehende und den Glaubenden gerecht erklärende, von der Sünde erlösende Gerechtigkeit verstanden wird. Gott erweist sie im Sühnetod Jesu. Die in der Forschung (keineswegs nur von Bultmann) vertretene These, dass Paulus in Röm 3,25 eine vorgeprägte Tradition verwendet, die eine eigenartige Vorstellung und Sprache hat, erachtet K. für nicht nötig. Dass die Wendung „durch den Glauben“ den Satzbau stört und eine paulinische Auslegung einer die Schicksalswende beschreibenden christologischen (liturgischen) Tradition sein kann, ist von Khalil nicht bedacht. Das ist schade. Denn die Suche nach liturgischen Traditionen im Neuen Testament dürfte der orthodoxen Theologie und Kirche durchaus entsprechen, schöpft doch die orthodoxe Theologie aus der Liturgie. Das könnte bei Paulus vergleichbar gewesen sein.

Michael G. Azar, „The Law and New Life in Rom 7:1-6. Eastern-Western Dialogue and Romans" (S. 243-271) untersucht die Aussagen eines Textes, der weniger mit Rechtfertigung, wohl aber viel mit dem Verständnis des Gesetzes durch Paulus zu tun hat. In einer sehr genauen Vers-fürVers-Auslegung stellt er heraus, dass der dem Gesetz abgestorbene Mensch in der Neuheit des Geistes zum Leben gerufen ist, das Gesetz aber nur in seinem buchstäblichen Sinn außer Kraft ist, nicht in seinem veränderten, vom Geist geprägten. Mit Blick auf die Dogmengeschichte betont er, dass die Kirche im Kampf gegen Markion an der Gültigkeit des alttestamentlichen Gesetzes als Richtschnur für den Christen festgehalten hat. Dafür führt er das eindrückliche Bild des Origenes an, wonach nach dem "Gesetz" die Witwe den Bruder des Verstorbenen heiraten soll. Der Christ ist also an 
den „Bruder“ des AT-Gesetzes gebunden, das geistlich verstandene „Gesetz“. Die Reformatoren hätten diesen östlichen Zugang zum Gesetz verlassen. Aber Melanchton und seine Schüler haben versucht, mit dem Patriarchen Jeremias II. Annäherungen zu erreichen. Das ist zwar nicht gelungen, aber in der reformierten Kirche ist der "tertius usus legis “ bedeutsam geworden, muss man hinzufügen, und ebenso, dass die „New Perspective on Paul“ diese Lehre voraussetzt.

James Buchanan Wallace, „Identities at Risk: «The New Perspective on Paul» and Eastern Orthodox Interpretation of Rom 8,14-17; 28-30" hebt die Tatsache hervor, dass der Geist bei der Taufe gegeben wurde, der von aller Furcht befreit. Der Abba-Ruf wird nicht als ekstatischer Schrei verstanden, sondern als Akzeptanz der geschenkten Sohnschaft. Diese Geistesgabe leitet einen geistlichen Prozess „von einer Herrlichkeit zur anderen“ (2 Kor 3,18) ein, die sich im Alltag konkretisieren muss. Der Geist wirkt mit unserem Geist zusammen. Die vorzeitliche Erwählung durch Gott gilt nicht separat dem Individuum für sich, sondern jedem Einzelnen in der Kirche.

Der Beitrag von Michael J. Gorman, „Reading Gal 2,15-21 Theologically. Beyond Old and New, Beyond West and East" (S. 321-347) ist der ungewöhnlichste in dieser Sammlung. Schon die Überschrift macht das deutlich: Er will nicht rein exegetisch, sondern theologisch den Text auslegen und das heißt, in seiner Bedeutsamkeit für uns heute. Seine Eigenwilligkeit zeigt sich bereits in der Übersetzung des Textes. Er versteht „Glauben Jesu“ als Treue (faithfullness) Jesu in Tod und Auferstehung, an der der Christ partizipieren kann. Er macht sein Konzept deutlich, indem er dem Text entlang geht. So versteht er Rechtfertigung (justification) als Eingliederung in Christus, als Teilhabe an seinem Tod und seiner Auferstehung und Verwandlung zur Liebe hin. Dies Verständnis hat sowohl forensische Bedeutung, als auch für den Bund Gottes mit seinem Volk. Damit hofft Gorman der alten wie der neuen „Perspective on Paul“ gleicherweise gerecht zu werden.

Der zweite Beitrag von Athanasios Despotis, „Beyond Theologiccal Arguments. The Ethics of Love and Coming to Faith in Paul“(S. 355-369) fällt aus dem Rahmen. Despotis geht es darum, das Verhältnis von Glaube und Liebe genauer zu bestimmen. Dazu bringt er zu Beginn eine Geschichte der Erforschung von Glaube und Liebe im NT. Sodann beschreibt er den Begriff des Glaubens zunächst als Annahme des Evangeliums und dann als Lebensgrundlage des Christen. Anschließend beschreibt er das Verständnis von Liebe und bemüht sich um den Nachweis, dass ein „um zum Glauben zu kommen" als Eingliederung in die Gemeinde, die Liebe Gottes zur Voraussetzung hat, die die Weitergabe der Liebe zu Gott und dem Mitmenschen veranlasst. Man hätte sich gewünscht, dass Despotis den Aorist episteusa als 
„zum Glauben kommen“ genauer betrachtet und davon ausgeht, dass agape ein nomen actionis ist.

Rikard Roitto, „Paul's theological Language of Salvation as Social and Embodied Cognition" (S. 377-399) zeigt auf, dass die theologische Sprache ein bestimmtes Verstehen wiedergibt, das durch die soziale Umwelt bedingt ist. Paulus hat sein Damaskuserlebnis meditiert, die Sprache dazu aber in der ihn gedanklich begleitenden Gemeinde gefunden. Dabei haben die Abendmahlsgemeinschaft und der Ritus der Taufe ihn besonders inspiriert. Als Zentrum der Theologie des Paulus sieht Roitto nicht die "Rechtfertigung" an, sondern die „Partizipation in Christus“, Rechtfertigung, Rettung und Erlösung gehören der Sprache des Übergangenges zu einer neuen Daseinsweise an. Sie sind alle als solche, wie auch die Aussage von der Verwandlung oder die Betonung der Theosis, gleich wichtig. Die gesamte theologische Sprache des Paulus ist bestimmt vom Durchgang zu der neuen Lebensweise „in Christus“, wobei die aktuellen Formulierungen von den bestimmten Gegebenheiten abhängen.

Im Rückblick auf diese einzigartige Sammlung von Studien zu einem zentralen Thema orthodoxer Theologie lässt sich zweierlei feststellen: Die meisten paulinischen Texte sind auch exegetisch eingehend behandelt, in ihren Einzelaussagen und innerhalb ihres Kontextes. Sowohl in exegetischer als auch systematischer Hinsicht wurde eine hohe Leistung vollbracht. Eine Untersuchung von Phil 3,2-11 fehlt. Sie war vorgesehen, ist aber ausgefallen. Sodann fällt auf, dass von den Kirchenvätern sehr oft Johannes Chrysostomos herangezogen wird. Das hängt wohl an dem Zugang zu den Quellen.

Trägt man die verschiedenen Beiträge zusammen und fragt nach dem Verständnis der Rechtfertigung durch Paulus, dann ergibt sich, dass für den Apostel mehrere sehr verschiedene Ereignisse zusammenfallen. Das sind: die Annahme der Botschaft des Evangeliums, also Glaube, die Taufe, die Gabe des Geistes, die Erklärung der Sohnschaft, die Eingliederung in Christus und damit in die Gemeinde, die Verwandlung des Menschen in eine neue Schöpfung und damit der Beginn eines neuen Weges der Heiligung, bei dem sogar Vollendung angestrebt werden kann. Für die folgenden Generationen, die bereits Kinder tauften, wurde der sakramentale Akt der Taufe stärker betont, die Botschaft des Evangeliums trat gegenüber der kirchlichen Lehre zurück, der persönliche Glaube wich einem Mitmachen in der Kirche. In der orthodoxen Theologie wurde der Weg der Heiligung als Weg zur Vollendung auf das Haupt hin (Eph 4,13) hervorgehoben. Dies führte zunächst zu einer Bezeichnung dieses Weges zum Ziel als Christosis und dann als Theosis. In Abwandlung von Gal 4,4 und 2 Kor 9,8 wurde gelehrt, dass 
Gott Mensch wurde, damit wir göttlich würden, gewiss in einem geistlichen Prozess. Die Reformatoren haben der Botschaft als Zuspruch und Anspruch ein erhöhtes Gewicht gegeben und den persönlichen Glauben hervorgehoben. Damit wurde aber der Weg der Heiligung in den Hintergrund gerückt. Die „New Perspective on Paul“ betont wieder den Weg der Heiligung und nähert sich damit dem jüdischen Verständnis des Gottesvolkes, das Paulus durch seine Abwehr der Gesetzeswerke nicht genügend berücksichtig hat. Der heutige Ausleger der paulinischen Botschaft empfindet so die Ausdeutungen der paulinischen Botschaft in den verschiedenen Kirchen als für die jeweilige Zeit und Umwelt der Sache entsprechend und dem Evangelium als Ganzes dienlich. 